\title{
Detecting Planets within Disks
}

\section{S. Wolf}

\author{
California Institute of Technology, 1201 E California Blvd, Mail code \\ 105-24, Pasadena, CA, 91125, USA; swolf@astro.caltech.edu
}

\begin{abstract}
Possible ways to detect extra-solar planets in circumstellar disks using high-angular resolution measurements (imaging) are discussed. Since dust is expected to outshine the planet by scattering of stellar radiation and thermal emission, the most promising approach is to look for characteristic signatures in the circumstellar disk caused by the interaction with the planet.
\end{abstract}

\section{Introduction}

Circumstellar disks are considered to be a natural outcome of the proto-stellar evolution, at least in the case of low to medium mass T Tauri and Herbig Ae/Be stars (e.g., Adams, Lada, \& Shu 1987). The disks provide the material and environment from and in which planets are expected to be formed (e.g., Lissauer 1993). Thus, if one wants to investigate how planets may be found in these disks one has to consider the evolution of both circumstellar disks and the planets therein. During the last two decades a detailed picture of the large-scale evolution of the circumstellar environment - in particular the circumstellar disk - has been worked out. The planet formation process, however, is in major parts still under discussion - mainly due to the lack of adequate constraints from observations. Thus, the search for planets in circumstellar disks is important in order to confirm or to rule out existing hypotheses on planet formation scenarios (see Sect. 2).

As it will be shown in Sect. 4, the direct detection of planets in circumstellar disks using high-resolution imaging techniques is expected to be extremely difficult (if at all feasible) both with existing telescopes and with observing equipment that will become available in the near future. However, numerical simulations show that planets may cause signatures in circumstellar disks that are in size much larger than the planet itself and thus much easier to detect. In Sect. 3 the most important of these signatures and their observability with the goal to use them as indicators and therefore as indirect hints of the presence of planets in disks are discussed.

\section{Imaging Precursors of Planet Formation}

There exist mainly two different pictures of planet formation. The first is characterized by the following phases: (1) Dust grain growth from submicron-sized particles to centimeter / decimeter-sized bodies via coagulation followed by (2) an 
agglomeration process that leads to the formation of (sub)kilometer-sized planetesimals which (3) by accretion form terrestrial (rocky) and Uranian (icy) planets or the cores of Jovian (gaseous) planets (e.g., Pollack et al. 1996, Weidenschilling 1997). Alternatively, planetesimals may form via the gravitational instability of solids that have settled to the midplane of a circumstellar disk (Goldreich \& Ward 1973, Youdin \& Shu 2002). Trying to trace these processes with imaging technique that are feasible nowadays or in the near future, one has to be aware that only scattering, absorption, and reemission of radiation by small dust grains (continuum) and gas absorption and emission (atomic/molecular lines) can be efficiently observed.

An example that underlines the importance of high-resolution imaging for tracing the earliest stage of planet formation are observations of the Butterfly star IRAS $04302+2247$ in Taurus. Using high-resolution near-infrared scattered light images of the circumstellar envelope obtained with the Hubble Space Telescope in combination with spatially resolved millimeter maps that trace the dust reemission from the circumstellar disk, different dust evolution scenarios in the environment of this young stellar object (Class I) have been revealed. Based on the comparison with radiative transfer simulations it has been shown that the dust grains in the circumstellar envelope of this source are still comparable with those of the interstellar medium, while the grains in the much denser circumstellar disk have grown by up to $2-3$ orders of magnitude (Wolf, Padgett, \& Stapelfeldt 2003 and references therein).

Another example that shows the possibility to detect large-scale density enhancements in circumstellar disks which may result from a disk instability has been discussed by Wolf \& Klahr (2003). In that particular study the possibility of mapping large-scale anticyclonic vortices, resulting from a global baroclinic instability as a precursor of planet formation in protoplanetary disks has been investigated. It was shown that the planned Atacama Large Millimeter Array (ALMA) will be able to trace these AU-sized vortices and will therefore allow testing of existing models of this very early stage of planet formation in circumstellar disks.

\section{Mapping Planet - Disk Interactions}

Once (proto-)planets have been formed, they may significantly alter the surface density profile of the disk and thus cause signatures in the disk that are much easier to find than the planets themselves. The appearance and type of these signatures depend on the mass and orbit of the planet, but even more on the evolutionary stage of the circumstellar disk: While the spatial structure of optically thick, young circumstellar disks around Herbig Ae/Be and T Tauri stars is dominated by gas dynamics, the much lower optical depth and gas-to-dust mass ratios in debris disks (Zuckermann, Forveille, \& Kastner 1995; Dent et al. 1995; Artymowicz 1997; Liseau \& Artymowicz 1998; Greaves, Coulson, \& Holland 2000) let the stellar radiation via Poynting-Robertson effect and stellar wind drag, in addition to gravitation, be responsible for the resulting disk density distribution. 

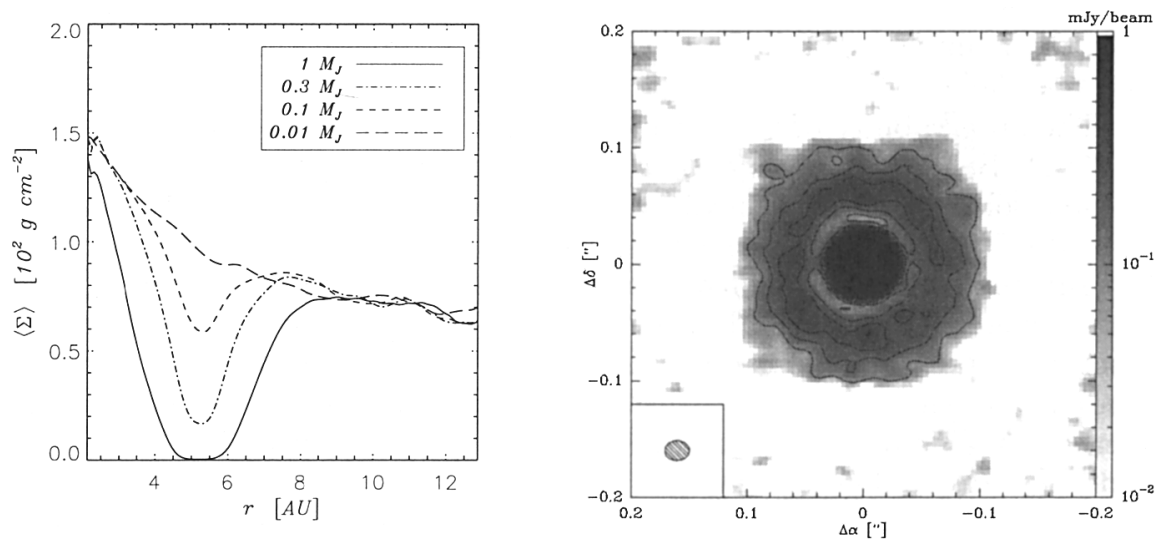

Figure 1. Left: Azimuthally averaged disk surface density for planets with masses of $0.01-1 \mathrm{M}_{\text {Jupiter }}$. The planet is located at a distance of $5.2 \mathrm{AU}$ from a solar-mass star. [G. D'Angelo, priv. comm.]. Right: Reconstructed image of a $0.05 \mathrm{M}_{\text {Sun }}$ circumstellar disk around a solar-type star with an embedded planet with a mass of $1 \mathrm{M}_{\text {Jupiter }}$ seen face-on, resulting from a simulation of ALMA. The gap at an angular distance of $37 \mathrm{mas}(5.2 \mathrm{AU})$ from the star is clearly visible. Wavelength: $700 \mu \mathrm{m}$ (428 GHz); bandwidth: $8 \mathrm{GHz}$; total integration time: $4 \mathrm{~h}$; system temperature: $500 \mathrm{~K}$; phase noise: $30^{\circ}$; max. baseline: $10 \mathrm{~km}$. [from Wolf et al. 2002].

\subsection{Young Circumstellar Disks}

Hydrodynamic simulations of gaseous, viscous protoplanetary disks with an embedded protoplanet show that the planet may open and maintain a significant gap (e.g., Bryden et al. 1999; Kley 1999, 2000). This gap, which is located along the orbit of the planet may extends up to several astronomical units in width, depending on the mass of the planet and the hydrodynamic properties of the disk. Nevertheless, the disk mass flow on to the planet continues through the gap with high efficiency. Nearly all of the flow through the gap is accreted by the planet at a rate comparable to the rate at which it would occur in the disk without a planet. The gas accretion on the planet can continue to planet masses of the order of $10 \mathrm{M}_{\text {Jupiter }}$, at which point tidal forces are sufficiently strong to prevent flow into the gap. However, planets with final masses of the order of $10 \mathrm{M}_{\text {Jupiter }}$ would undergo large migration, which makes formation and survival difficult. The simulations also show that only planets with masses $\gtrsim 0.1 \mathrm{M}_{\text {Jupiter }}$ produce significant perturbations in the disk's surface density (Bate et al. 2003; Fig. 1).

Protoplanets also launch spiral waves. However, in the presence of turbulence, these waves appear to be diffused and dissipated (Nelson \& Papaloizou 2003). Furthermore, D'Angelo, Henning, \& Kley (2002) found smaller-scale spirals in the vicinity of the planet that are detached from the main ones: Along these 
small spirals the gas orbits the planet, representing a circumplanetary disk (see also Lubow, Seibert, \& Artymowicz 1999). In contrast to this, the material along the main spirals follows the disk rotation around the star, moving away from the planet.

The observability of the outlined features has been investigated by Wolf et al. (2002). Based on hydrodynamic simulations of a $0.05 \mathrm{M}_{\text {Sun }}$ circumstellar disk around a solar-type star with an embedded planet of a mass of $1 \mathrm{M}_{\text {Jupiter }}$, images of the disk have been calculated. It was shown, that high-resolution images in the (sub-)millimeter wavelength range as to be obtained with the millimeter interferometer ALMA will allow to trace the gap, but not the large-scale spiral structure or the circumplanetary disk (see Fig. 1). At shorter, e.g., near to midinfrared wavelengths, however, the extreme brightness contrast between the hot innermost region of the disk and the gap located in a Jupiter-like orbit makes it hardly feasible to detect the gap.

Since the location, depth, and width of a gap depends on the mass and orbital parameters of the planet, these parameters can be derived from highresolution images, once they are available. However, one should be aware, that these interpretations will rely on the comparison with high-quality simulations and thus on the physical processes considered. For example, the results from magneto-hydrodynamic simulations of circumstellar disks with an embedded planet show, that the gaps are shallower and asymmetrically wider than in case of pure hydrodynamic simulations, and the rate of gap-formation is slowed (Winter, Balbus, \& Hawley 2003; see also Nelson \& Papaloizou 2003).

\subsection{Debris Disks}

In evolved, optically thin debris disks, a planet produces (1) an asymmetric resonant dust belt with one or more clumps, intermittent with one or a few off-center cavities, and (2) a central cavity void of dust via resonances and gravitational scattering (Ozernoy et al. 2000, Liou \& Zook 1999). The resulting characteristic density patterns are expected to provide the strongest indirect hints on the existence of planets in these disks (e.g., Ozernoy et al. 2000 - Fig. 1). Kuchner $\&$ Holman (2003) pointed out that four basic resonant structures probably represent the range of high-contrast resonant structures a planet with eccentricity $\lesssim 0.6$ can create in a disk of dust released on low eccentric orbits: (i) A ring with a gap at the location of the planet, (ii) a smooth ring, (iii) a clumpy eccentric ring, and (iv) an offset ring plus a pair of clumps. The appearance / dominance of one of these structures mainly depends on the mass of the planet and the eccentricity of its orbit (see Fig. 2).

Indeed, several debris disks around nearby main-sequence stars show structures and asymmetries that are considered to result from planetary perturbations (Holland et al. 1998, 2003; Schneider et al. 1999; Koerner, Sargent, \& Ostroff 2001). A prominent example is the debris disk around Vega which shows two dominating emission peaks / density enhancements (Holland et al. 1998, Wilner et al. 2002). However, images tracing the thermal dust reemission with higher spatial resolution are required to verify this hypothesis.

Besides high-resolution imaging of debris disks, mid-infrared spectroscopy is a valuable tool to deduce the existence of an inner gap, since the deficiency of hot dust in the stellar vicinity causes a decrease of the mid-infrared flux compared 

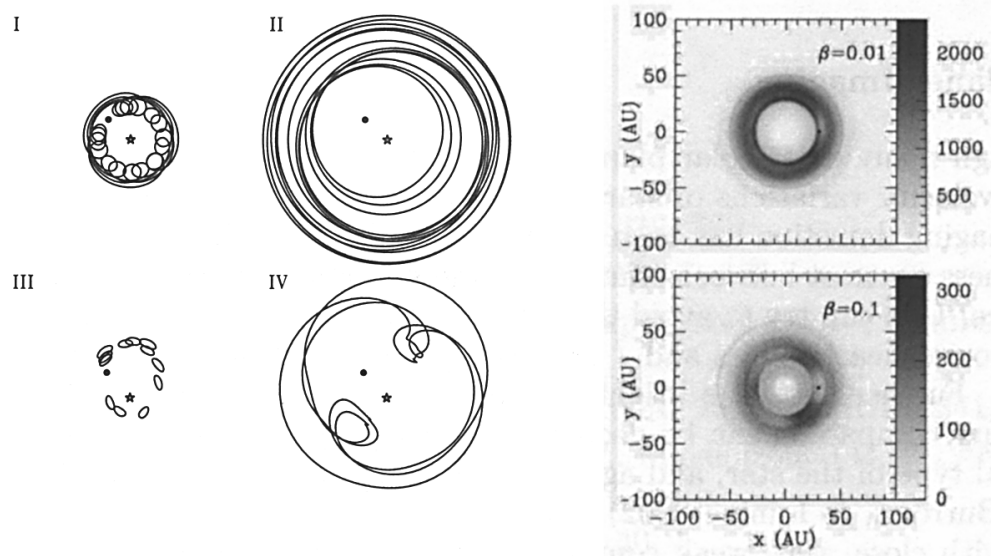

Figure 2. Left: Four basic resonant structures in debris disks: (I) low-mass planet on a low-eccentricity orbit, (II) high-mass planet on a low-eccentricity orbit, (III) low-mass planet on a moderate-eccentricity orbit, and (IV) high-mass planet on a moderate-eccentricity orbit [from Kuchner \& Holman 2003]. Right: Simulated particle number density distribution in the solar system (Mercury and Pluto are excluded). The parent bodies which create small dust grains by collisions are randomly distributed in the ranges 35-50 AU (symbolizing the Kuiper Belt; $i=$ $\left.0^{\circ}-17^{\circ}\right)$. Two different particle sizes are considered, indicated by the ratio of the radiation pressure to gravity $\beta$ (Burns et al. 1979; for grains consisting of amorphous Olivine, $\mathrm{MgFeSiO}_{4}, \beta=0.01 / 0.1$ corresponds to dust grain radii of $13 \mu \mathrm{m} / 1.8 \mu \mathrm{m})$. The dot at $(30,0)$ indicates the position of Neptune [from Moro-Martín \& Malhotra 2002; see also Liou \& Zook 1999].

to an undisturbed disk. In several prominent debris disks, inner cavities have been found: $\beta$ Pic (inner radius: $20 \mathrm{AU}), \mathrm{HR} 4796 \mathrm{~A}(30-50 \mathrm{AU}), \epsilon$ Eri $(50 \mathrm{AU})$, Vega (80 AU), and Fomalhaut (125 AU) - see, e.g., Dent et al. 2000; Greaves, Mannings, \& Holland 2000; Wilner et al. 2002; Holland et al. 2003. But also in case of T Tauri disks inner cavities have been found that are much larger than determined by the dust sublimation temperature: GM Aurigae (Koerner, Sargent, \& Beckwith 1993; Rice et al. 2003) and TW Hya (Calvet et al. 2002).

Another example in case of which the possible influence of a planet on the disk structure has been discussed is the $\beta$ Pictoris disk. The dust disk, seen nearly edge-on, extends to at least a distance of $100 \mathrm{AU}$ from the central star (Zuckerman \& Becklin 1993, Holland et al. 1998, Dent et al. 2000, Pantin, Lagage, \& Artymowicz 1997). The Northeast and Southwest extensions of the dust disk have been found to be asymmetric in scattered light as well as in 
thermal emission. This warp is assumed to be caused by a giant planet on an inclined orbit that gravitationally perturbs the dust disk (Augereau et al. 2001, Mouillet et al. 1997; see also Lubow \& Ogilvie 2001).

\section{Planet Imaging}

Although many extra-solar planet candidates have been detected indirectly by radial velocity variations of stars (e.g., review by Marcy \& Butler 2000) no direct imaging detection has been obtained so far. The main difficulty is the large brightness contrast between stars and planets combined with their small angular distance. One can try to avoid this problem by searching for planetary companions around nearby stars and / or making use of high-resolution imaging techniques. Furthermore, the brightness ratio between the star and the presumed planetary companion can be decreased by choosing a proper wavelength range, spectral type of the star, and age / evolutionary stage of the planet (e.g., Hubbard, Burrows, \& Lunine 2002). Indeed, recent observations of main-sequence stars with close, very weak companions show that direct imaging detection of (giant) extra-solar planets is possible with current technology (e.g., Potter et al. 2002, König et al. 2002)

To image planets which are still embedded in a circumstellar disk is even more difficult, because the dust continuum radiation dominates the whole spectral range from the ultraviolet to millimeter wavelengths: In the ultraviolet to the (near-) infrared by scattering of the stellar radiation and at longer wavelengths by thermal reemission. Thus, in the preparation of observations of planets in disks even more constraints have to be taken into account, describing the properties of the disk: the optical parameters of the dust (chemistry, size distribution) and the spatial temperature and density distribution of the dust. In case of young optically thick circumstellar disks, the disk inclination and possible accretion on the planet have to be considered as well.

\section{Summary}

Numerical simulations convincingly demonstrate that high-resolution imaging performed with instruments / telescopes that are or will become available in the near future will allow detection of planets in circumstellar disks. However, scattering of stellar light and thermal emission by small grains (in the case of optically thick disks seen at high inclination also absorption) will make direct planet detection via imaging hardly feasible. But planets cause large-scale perturbations in the disks which will be observable with observatories like ALMA, SOFIA $^{1}$, and JWST $^{2}$ by mapping the dust reemission. The particular type of perturbation depends on the evolutionary stage of the disk. Primary signatures of planets embedded in disks are gaps in case of young disks and characteristic asymmetric density patterns in debris disks. Secondary signatures are, for

\footnotetext{
${ }^{1}$ Stratospheric Observatory For Infrared Astronomy

${ }^{2}$ James Webb Space Telescope
} 
instance, spiral structures in young disks or warps due to planets on inclined orbits.

Acknowledgments. This study was supported by NASA through grant NAG 5-11465.

\section{References}

Adams, F.C., Lada, C.J., Shu, F.H. 1987, ApJ, 312, 788

Artymowicz, Annu. Rev. Earth Planet. Sci. 1997, 25, 175

Augereau, J.C., Nelson, R.P., Lagrange A.M., Papaloizou, J.C.B., Mouillet 2001, A\&A, 370, 447

Bate, M.R., Lubow, S.H., Ogilvie, G.I., Miller, K.A. 2003, MNRAS, 341, 213

Bryden, G., Chen, X., Lin, D.N.C., Nelson, R.P., Papaloizou, J.C.B. 1999, ApJ, 514,344

Burns, J.A., Lamy, P.L., Soter, S. 1979, Icarus, 40, 1

Calvet, N., D’Alessio, P., Hartmann, L., Wilner, D., Walsh, A., Sitko, M. 2002, ApJ, 568, 1008

D'Angelo, G.D., Henning, Th., Kley, W. 2002, A\&A, 385, 647

Dent, W.R.F., Greaves, J.S., Mannings, V., Coulson, I.M., Walther, D.M. 1995, MNRAS, 277, L25

Dent, W.R.F., Walker, H.J., Holland, W.S., Greaves, J.S. 2000, MNRAS, 314, 702

Goldreich, P., Ward W.R. 1973, ApJ, 183, 1051

Greaves, J.S., Coulson, I.M., Holland, W.S. 2000, MNRAS, 312, L1

Greaves, J.S., Mannings, V., Holland, W.S. 2000, Icarus, 155

Holland, W.S., Greaves, J.S., Dent, W.R.F., Wyatt, M.C., Zuckerman, B., et al. 2003, ApJ, 582, 1141

Holland, W.S., Greaves, J.S., Zuckerman, B., et al. 1998, Nature, 392, 788

Hubbard, W.B., Burrows, A., Lunine, J.I. 2002, ARA\&A, 40, 103

Kley, W. 1999, MNRAS, 303, 696

Kley, W. 2000, MNRAS, 313, L47

Koerner, D.W., Sarget, A.I., Beckwith, S.V.W. 1993, Icarus, 106, 2

Koerner, D.W., Sargent, A.I., Ostroff, N. 2001, ApJ, 560, L181

König, B., Fuhrmann, K., Neuhäuser, R., Charbonneau, D., Jayawardhana, R. 2002, A\&A, 394, L43

Kuchner, M.J., Holman, M.J. 2003, ApJ, 588, 1110

Liou, J.-C., Zook, H.A., 1999, AJ, 118, 580

Liseau, R., Artymowicz, P. 1998, A\&A, 334, 935

Lissauer, J. J. 1993, ARA\&A, 31, 129

Lubow, S.H., Ogilvie, G.I 2001, ApJ, 560, 997

Lubow, S.H., Seibert, M., Artymowicz, P. 1999, ApJ, 526, 1001

Marcy, G.W., Butler, R.P., 2000, PASP, 112, 137 
Moro-Martín, A., Malhotra, R. 2002, ApJ, 124, 2305

Mouillet, D., Larwood, J.D., Papaloizou, J.C.B., Lagrange, A.M. 1997, MNRAS, 292, 896

Nelson, R.P., Papaloizou, J.C.B. 2003, MNRAS, 339, 993

Ozernoy, L.M., Gorkavyi, N.N., Mather, J.C., Taidakova, T.A. 2000, ApJ, 537, L147

Pantin, E., Lagage, P.-O., Artymowicz, P. 1997, A\&A, 327, 1123

Pollack, J.B., Hubickyj, O., Bodenheimer, P., Lissauer, J.J., Podolak, M., Greenzweig, Y. 1996, Icarus, 124, 62

Potter, D., Martín, E.L., Cushing, M.C., Baudoz, P., Brandner, W., Guyon, O., Neuhäuser, R. 2002, ApJ, 567, L133

Rice, W.K.M., Wood, K., Armitage, P.J., Whitney, B.A., Bjorkman, J.E. 2003, MNRAS, 342,79

Schneider, G., Smith, B.A., Becklin, E.E. 1999, ApJ, 513, L127

Weidenschilling, S. 1997, Icarus, 127, 290

Wilner, D.J., Holman, M.J., Kuchner, M.J., Ho, P.T.P. 2002, ApJ, 569, L115

Winters, W.F., Balbus, S.A., Hawley, J.F. 2003, ApJ, 589, 543

Wolf, S., Gueth, F., Henning, Th., Kley, W. 2002, ApJ, 566, L97

Wolf, S., Klahr, H. 2003, ApJ, 2002, ApJ 578, L79

Wolf, S., Padgett, D.L., Stapelfeldt, K.R. 2003, ApJ, 588, 373

Youdin, A.N., Shu, F.H. 2002, ApJ, 580,494

Zuckerman, B., Becklin, E.E. 1993, ApJ, 414, 793

Zuckermann, B., Forveille, T., Kastner, J.H. 1995, Nature, 373, 494 